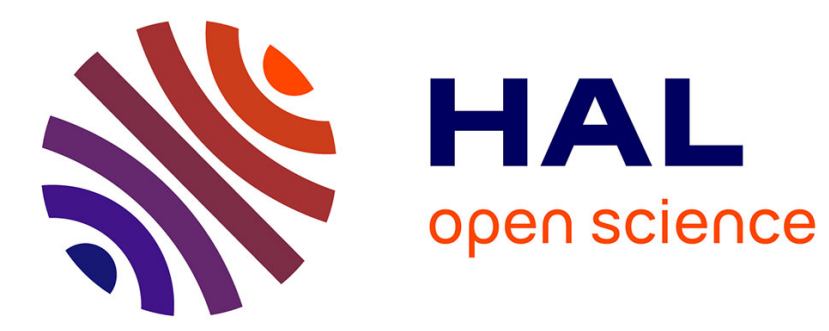

\title{
Traffic shaping in wireless home networks
}

Pirathayini Srikantha, Marianna Carrera, Martin May

\section{To cite this version:}

Pirathayini Srikantha, Marianna Carrera, Martin May. Traffic shaping in wireless home networks. SIGMOBILE Mob. Comput. Commun. Rev., 2010, 14 (4), pp.52-54. 10.1145/1942268.1942287. hal-00597416

\section{HAL Id: hal-00597416 https://hal.science/hal-00597416}

Submitted on 31 May 2013

HAL is a multi-disciplinary open access archive for the deposit and dissemination of scientific research documents, whether they are published or not. The documents may come from teaching and research institutions in France or abroad, or from public or private research centers.
L'archive ouverte pluridisciplinaire HAL, est destinée au dépôt et à la diffusion de documents scientifiques de niveau recherche, publiés ou non, émanant des établissements d'enseignement et de recherche français ou étrangers, des laboratoires publics ou privés. 


\title{
MobiCom 2010 Poster: \\ Traffic Shaping in Wireless Home Networks.
}

\author{
Pirathayini Srikantha ${ }^{a b}$ \\ psrikant@engmail.uwaterloo.ca \\ Marianna Carrera ${ }^{a c}$ \\ Martin May ${ }^{a}$ \\ ${ }^{a}$ Technicolor, ${ }^{b}$ University of Waterloo, ${ }^{c}$ UPMC Sorbonne Universités
}

\begin{abstract}
Many devices commonly found in wireless home networks such as smartphones, gaming consoles and media servers generate real-time traffic with stringent performance requirements. Since the wireless medium is mostly accessed in a distributed manner (DCF), sensitive traffic needs to be "protected". This work investigates how mechanisms defined in the WiFi standard such as virtual carrier sensing and CTS frames can enable this without requiring any modifications to end devices. $n s-3$ simulations of this scheme in typical wireless home network configurations show significant performance improvement in the delivery rate of prioritized nodes compared to those using plain DCF.
\end{abstract}

\section{Introduction}

A typical wireless home network can contain nodes that generate a wide variety of traffic. One specific example of such a network is illustrated in Figure 1. In this network, devices such as the media server and IPTV, exclusively generate or receive real-time traffic with stringent bandwidth requirements. Such traffic can be severely impaired by other traffic as the bandwidth available to a particular device depends on how the medium is used by other stations.

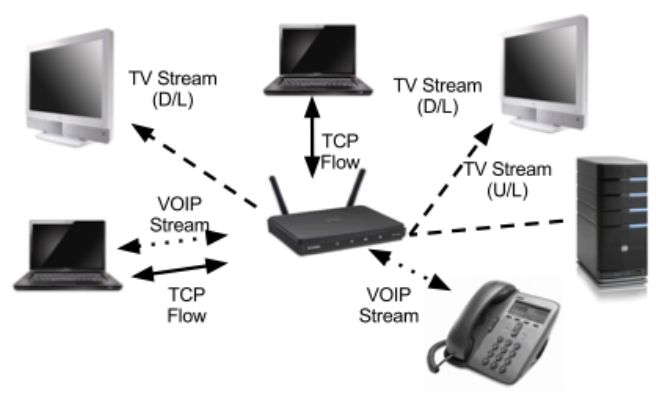

Figure 1: Example of a Typical Home Network

Wireless nodes, in today's networks, mostly access the medium using IEEE 802.11's DCF protocol. Since there is no notion of per-station priority in DCF, all devices will equally contend for the channel. In order to protect nodes with sensitive traffic, $\mathrm{PCF}$ and $\mathrm{HCF}$ were standardized [1]. To the best of our knowledge, consumer devices and applications have not yet embraced these standards.

The goal of our work is to enable protection of sensitive traffic without having to modify end devices. We intend to capitalize on mandatory mechanisms defined in the IEEE 802.11 standard for this purpose. Our proposal in this paper only requires modification to the AP - no end devices will require changes.

\section{Our Approach}

Our solution consists of a central controller that will serve as the coordination point of the wireless network - making decisions on how the shared medium is accessed by stations in the wireless home network. In our design, the AP assumes the role of central controller as it has a holistic view of its BSS. The AP will allocate priority to nodes associated to the same BSS according to a scheduling algorithm. The focus of this extended abstract is not the scheduling algorithm but rather the medium reservation mechanism.

In order to centrally control medium access in the presence of legacy stations which typically access medium in a distributed manner (DCF), we adapt a technique similar to that defined in Virtual PCF [2]. This technique was used in [2] to schedule VoIP traffic and we extend it to general traffic types.

Traffic in a wireless home network aggregates at the AP in both uplink and downlink directions. The AP will transmit downlink frames by accessing the medium aggressively, i.e., with shorter interframe times than regular stations using DCF. To protect a station with an uplink frame, the AP will access the medium aggressively to transmit it an unsolicited CTS frame. All stations, except for the destination of the CTS frame, will remain silent for the time specified in the duration field of the CTS frame. During this period, since only the protected station will be allowed to contend for medium access, our mechanism results in implicit polling.

Next we define our proposed system's parameters. Consider a wireless home network consisting of one AP and $n$ stations, of which $p$ stations have to be protected. Let $t$ represent the total time reserved by all the CTS frames transmitted by the AP in a reference time interval $T$. A station $i \in p$ is allocated a fraction $w_{i}$ of $t$ for prioritized access to the medium. This fraction is determined by the following relation:

$$
w_{i}=\frac{\sum_{j=1}^{C_{i}} D_{j}^{i}}{t}, t=x T, x \in[0,1],
$$

where, $C_{i}$ represents the total number of CTS frames 
transmitted by the AP to station $i$ in the reference time interval $T$; and $D_{j}^{i}$ is duration field of the $j^{\text {th }}$ CTS frame transmitted to node $i$. Free variables of this problem are $w_{i}, C_{i}, D_{j}^{i}, t$ and $T$.

In the following sections we evaluate the impact of varying $D_{j}^{i}$ (which remains constant for all $j$ ) on the effectiveness of medium reservation for uplink traffic.

\section{Simulation Settings}

The simulated wireless network is an infrastructure BSS consisting of a single AP and multiple wireless nodes. All nodes in the simulations use IEEE 802.11a standard for medium access. We use the following models to enable realism in the simulations: Log Distance Propagation Loss Model, Constant Speed Propagation Delay Model, SINR Interference Model and Constant Rate Adaptation (6 Mbps data rate).

Only the simulator core's AP module has been modified to accommodate transmissions of unsolicited CTS frames. Modules belonging to wireless stations have not been modified. Each simulation step lasts for 10 seconds (stability has been tested). In all simulations presented below, within each simulation step $D_{j}^{i}$ is constant, while across simulation steps the value of $D_{j}^{i}$ is incremented by $100 \mu s$.

We present results on two network topologies: in one configuration (CS) the stations are able to carrier sense one another and in the other (HT) the stations cannot carrier sense each other causing hidden terminal collisions at the AP.

\section{Study of the medium reservation}

In this paper, we study the overhead and effectiveness of the medium reservation mechanism. We define delivery rate $(d r)$ to be the amount of data received by the AP from a station over a simulation interval - which in this paper is 10 seconds. The delivery rate obtained using plain DCF under the same settings will serve as benchmark for the overhead study. For the effectiveness study, we compare the $d r$ of protected stations against that of non-protected ones. Nodes in all simulations will have backlogged queues i.e., will always have frames for transmission.

\section{IV.A. Medium reservation overhead}

Unsolicited CTS frames used to implicitly poll protected stations occupy the medium for the period required for their transmission. In order to compare the protocol overhead of the CTS-based medium access mechanism with respect to plain DCF, we compare the delivery rates obtained by a single station $A(n=1)$ always having frames to transmit, with and without the CTS protection mechanism. Station A transmits frames with a payload size of 1000 bytes for the experiments in Figure 2 and variable payload sizes for experiments in Figure 3.
As station A always has frames to transmit with no other nodes competing with it, the delivery rate obtained using regular DCF for medium access serves as the upper bound. We compare this upperbound with the delivery rate obtained by $A$ when the AP reserves the medium entirely to the link from $A$ to the AP: $w_{A}=1, x=1$, i.e., the AP transmits a CTS frame to $A$ as soon as the medium is free.

We observe in Figure 2 that the delivery rate increases (i.e., the overhead decreases) when the reservation duration $\left(D_{j}^{A}\right)$ is a multiple of the time required to transmit an uplink frame, and remains stable in between these periods. These increases can be observed when the proportion of CTS frames to uplink frames decreases.

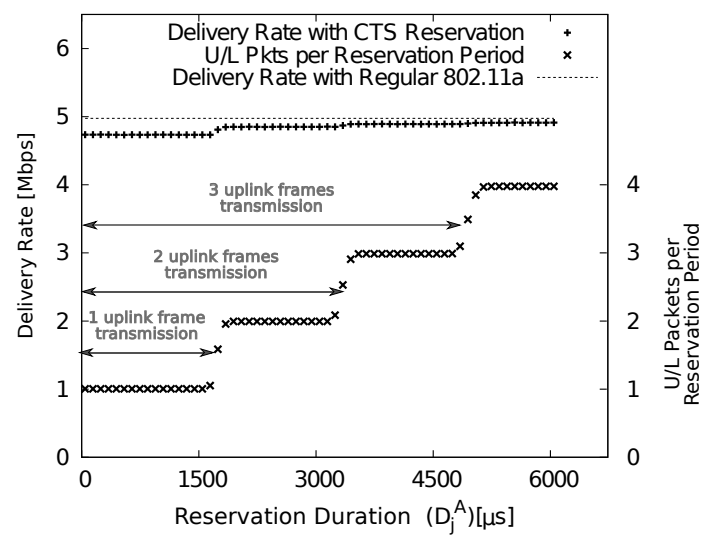

Figure 2: Effect of Batching Frames on Delivery Rate Overhead

Figure 3 illustrates the effect of varying payload sizes on the delivery rate overhead. To ease comparison, we show the difference between delivery rates obtained with regular DCF and that with our scheme, for every payload size. For smaller data payloads and reservation durations, the overhead is higher as more CTS frames are transmitted to reserve the medium for every frame.

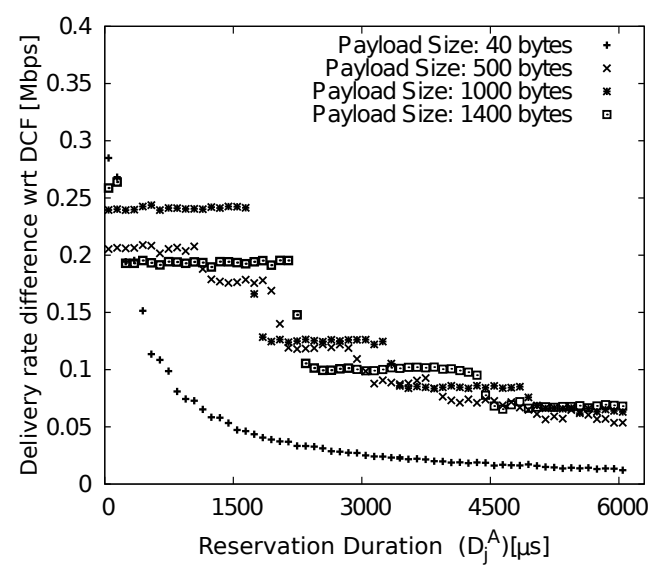

Figure 3: Effect of Data Payload Size on Delivery Rate Overhead

We conclude that the overhead even for small payloads and reservation durations, is not very significant (around 6\%) for a data rate of $6 \mathrm{Mbps}$. Furthermore, 
this overhead can be reduced by using large payload sizes or batching multiple frames into a single reservation. Video streaming, even live IPTV, typically use large buffers, and Layer-2 frames are normally MTUsized - hence this confirms that our approach is suited for real-time traffic.

\section{IV.B. Medium reservation effectiveness}

This section investigates how reservation duration can impact the manner in which stations heed the unsolicited CTS frames transmitted by the AP.

We now consider two stations $(n=2), A$ and $B$ which generate frames with a fixed payload size of 1000 bytes. We consider two extreme reservation policies: in one case only the link from $A$ to the AP is protected and is granted full share of the medium $\left(w_{A}=1, x=1\right)$; in the second case both stations are protected and are granted equal portions of the medium $\left(w_{A}=w_{B}=0.5, x=1\right)$. In the latter case, a CTS frame is addressed to each station in an alternating manner -i.e., in a round robin fashion.

Carrier Sensing Topology In this scenario, station $A$ and station $B$ carrier sense each other. Figure 4 shows that the delivery rate of station $\mathrm{A}$, when granted the entire medium, is close to that of $A$ transmitting alone using plain DCF. Moreover, the delivery rate of A using this scheme is more (as packet collisions are avoided) than twice that of $A$ competing with $B$ without the CTS reservation scheme. Station $B$ is deprived of access to the medium when the AP grants exclusive access to station $A$. When round robin scheduling is used, as expected, both nodes' delivery rates becomes half that of Node A with exclusive scheduling.

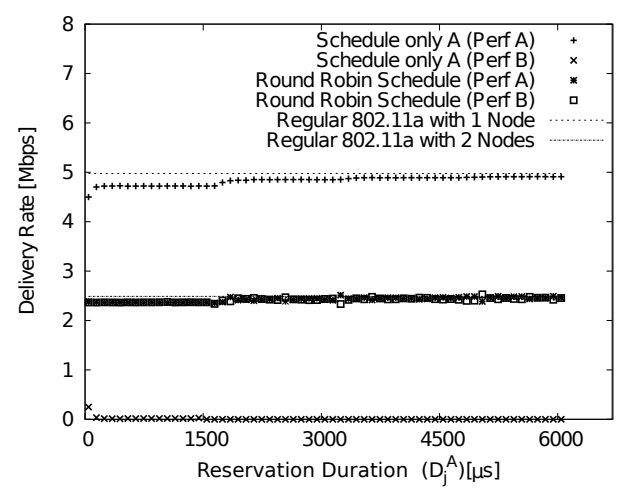

Figure 4: Nodes in CS Range - Round Robin and Exclusive Scheduling

Figure 4 also shows that it is not necessary to set the reservation duration to the time required to transmit an entire frame. Since nodes can sense each other's transmission, $D_{j}^{i}$ only needs to be large enough to allow the scheduled node to start frame transmission. Once the transmission begins, all other nodes will be able to hear it and remain silent. Values as small as 44 $\mu s$ in this setting is sufficient for the scheduled node to capture the channel. In the network topology used for this experiment, the CTS frames' duration field does not seem to affect the effectiveness of the reservation mechanism, but only the mechanism overhead.

Hidden Terminal Topology We now repeat the evaluation described above in a scenario where stations $A$ and $B$ are hidden from each other. All other settings remain the same. When using regular DCF, $A$ and $B$ will almost systematically collide at the AP. We now study the performance of the CTS-based reservation mechanism in this scenario and the effect of varying the reservation duration.

Figure 5 shows that when the AP shares the medium evenly between stations $A$ and $B$, their delivery rate is higher than that obtained with plain DCF for any reservation time. This is expected since CTS frames have been introduced by the standard along with RTS frames to solve hidden terminal problems.

Highest delivery rates are achieved when the reservation duration covers the transmission of one or multiple frames. Collisions are avoided since nodes are prevented from transmitting during the entire duration of an ongoing transmission.

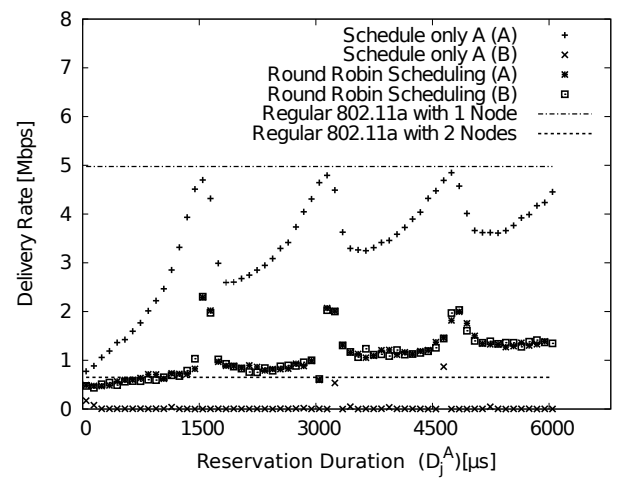

Figure 5: Nodes in HT Range.

\section{Conclusions}

From the simulations above, the following key observations are made of the CTS reservation scheme: it does not cause a large overhead, it succeeds in reserving the channel for scheduled nodes without requiring any modifications at the end nodes, the reservation duration needs to be large enough to give a head start for the frame transmission in the CS case and the reservation duration needs to be large enough to transmit an entire frame in the HT case.

As future work, we will extend the analysis in this work to the following instances: nodes having realistic traffic and variable data rates. Ultimately, a dynamic on-line scheduling algorithm making use of these analyses should be designed so that nodes with sensitive traffic can be protected.

\section{References}

[1] IEEE Computer Society, "Standard 802 Part 11," 2007.

[2] U. Ismail, "Virtual PCF: Improving VoIP over WLAN performance with legacy clients," Master's thesis, University of Waterloo, June 2009. 